\title{
All-Digital Massive MIMO with a Fronthaul Constraint
}

\section{Conference Paper}

\section{Author(s):}

Jacobsson, Sven; Ettefagh, Yasaman; Durisi, Giuseppe; Studer, Christoph (D)

Publication date:

2018-06

Permanent link:

https://doi.org/10.3929/ethz-b-000460181

Rights / license:

In Copyright - Non-Commercial Use Permitted

Originally published in:

https://doi.org/10.1109/SSP.2018.8450779 


\title{
ALL-DIGITAL MASSIVE MIMO WITH A FRONTHAUL CONSTRAINT
}

\author{
Sven Jacobsson ${ }^{1,2}$, Yasaman Ettefagh ${ }^{2}$, Giuseppe Durisi ${ }^{2}$, and Christoph Studer ${ }^{3}$ \\ ${ }^{1}$ Ericsson Research, Gothenburg, Sweden; ${ }^{2}$ Chalmers University of Technology, Gothenburg, Sweden; \\ ${ }^{3}$ Cornell University, Ithaca, NY, USA
}

\begin{abstract}
We analyze the uplink and downlink rates achievable in an all-digital massive multiple-input multiple-output system in which the base station (BS) is equipped with low-precision analog-to-digital and digital-to-analog converters, the size of the antenna array is limited, and there is a bandwidth constraint on the fronthaul link connecting the remote radio head to the digital baseband processing unit at the BS. Our results show that, at low SNR, it is better to use antenna arrays with many antenna elements, each one connected to low-precision converters, because this yields a beneficial array gain. On the contrary, at high SNR, it is better to reduce the number of active antenna elements, but increase the precision of the converters connected to them, because this allows one to separate more easily the data streams of the different users with simple linear baseband processing techniques.
\end{abstract}

Index Terms - massive MIMO, fronthaul, ADC, DAC

\section{INTRODUCTION}

All-digital massive multiple-input multiple-output (MIMO) beamforming architectures are superior in terms of performance and reconfigurability to hybrid analog-digital solutions. Such architectures, however, require the transfer of an excessively high amount of data on the fronthaul link, which connects the baseband unit (BBU) carrying out digital baseband processing, with the remote radio head (RRH) containing all radio-frequency circuitry, such as mixers, filters, power amplifiers, and analog-to-digital and digital-to-analog converters (ADCs and DACs). A promising approach to reduce the fronthaul bottleneck in all-digital architectures is to reduce the precision of the converters at the RRH.

In this paper, we investigate how the uplink and downlink rates of an all-digital massive MIMO system with limited fronthaul bandwidth depend on the number of base station (BS) antennas and on the precision of the ADCs and the DACs. To this end, we present achievable rate expressions

\footnotetext{
The work of SJ, YE, and GD was supported in part by the Swedish Foundation for Strategic Research under grant ID14-0022, and by the Swedish Governmental Agency for Innovation Systems (VINNOVA) within the competence center ChaseOn. The work of CS was supported in part by Xilinx, Inc. and by the US NSF under grants ECCS-1408006, CCF-1535897, CCF1652065, and CNS-1717559.
}

and perform numerical simulations under line-of-sight (LOS) conditions with a uniform linear array (ULA) of fixed size.

The uplink rates achievable over frequency-flat channels for BSs with low-precision ADCs and linear processing have been analyzed in, e.g., [1-4]. More sophisticated detection schemes, tailored to the nonlinearity introduced by low-precision ADCs, have been studied in [5,6]. Furthermore, the frequency-selective case and the use of orthogonal frequency-division multiplexing have been discussed in, e.g., [7,8]. All these results indicate that high achievable rates are supported even with 1-bit ADCs. Furthermore, lowprecision ADCs (e.g., with 3-5 bits) are sufficient to approach infinite-precision performance.

The downlink rates achievable over frequency-flat channels for BSs with low-precision DACs and linear precoding have been analyzed in [9-11]. More sophisticated precoding schemes, which outperform linear precoders at the cost of increased complexity, have been developed in, e.g., [11-16]. An extension of these results to frequency-selective channels have been discussed in [16-18]. As for the uplink, these results indicate that low-precision DACs (e.g., with 3-5 bits) are sufficient to approach infinite-precision performance.

All the results reviewed so far do not consider any fronthaul bandwidth constraints in their analysis. Fronthaulconstrained communication and fronthaul compression are well-studied subjects, especially in the context of cloud radio access networks $[19,20]$. In contrast to this line of work, we focus in this paper on a simple architecture in which the RRH has no digital signal-processing capabilities, and the fronthaul link between the RRH and the BBU carries uncompressed data samples.

\section{SYSTEM MODEL}

We consider the massive MIMO BS architecture in Fig. 1. We focus on a single-cell scenario in which the $B$-antenna BS communicates with $U$ single-antenna user equipments (UEs) in the same time-frequency resource. Uplink and downlink transmissions are separated via time-division duplexing.

We assume that each antenna is connected to a pair of identical ADCs and DACs, one for the in-phase (I) and one for the quadrature $(\mathrm{Q})$ component of the baseband signal. Let $Q$ denote the precision (measured in the number 


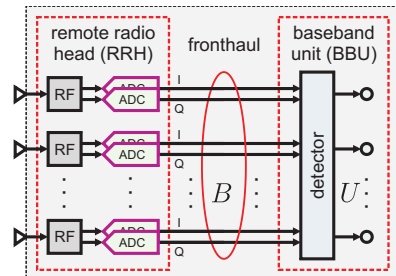

(a) Uplink: the RRH quantizes the received signal using $2 B Q$-bit ADCs; the quantized I/Q data are sent to the BBU over the bandwidthconstrained fronthaul link.

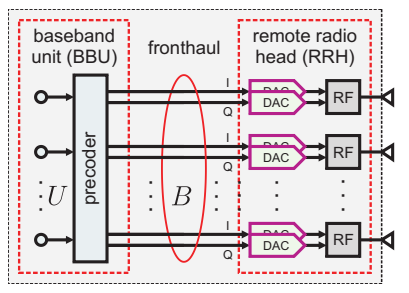

(b) Downlink: the BBU quantizes the precoded vector to match the precision of the $2 B Q$-bit DACs at the RRH; the I/Q data are sent to the RRH over the bandwidthconstrained fronthaul link.
Fig. 1. Overview of the all-digital $B$-antenna BS architecture. The BS is split into two units: the baseband unit (BBU) and the remote radio-head (RRH), which are connected via a bandwidth-constrained fronthaul link.

of bits) of the ADCs and DACs. Then, the fronthaul rate required for exchanging data between RRH and BBU (ignoring control and synchronization overhead and assuming uncompressed transmission) is

$$
R^{\mathrm{fh}}=2 B Q \quad[\mathrm{bit} / \mathrm{s} / \mathrm{Hz}] .
$$

We assume for simplicity that the ADCs and the DACs at the BS operate at Nyquist rate (i.e., no oversampling) and that they are equipped with ideal anti-aliasing and reconstruction filters, respectively. Furthermore, we assume that the BS has perfect channel state information (CSI) and that the data converters at the UEs are ideal, i.e., they have infinite precision.

We assume that all quantizers at the BS are uniform and symmetric. Specifically, if $r \in \mathbb{R}$ is the input to a quantizer, its output is

$$
\mathcal{Q}(r)= \begin{cases}\frac{\Delta}{2}\left(1-2^{Q}\right) & \text { if } r<-\frac{\Delta}{2} 2^{Q} \\ \Delta\left\lfloor\frac{r}{\Delta}\right\rfloor+\frac{\Delta}{2} & \text { if }-\frac{\Delta}{2} 2^{Q} \leq r<\frac{\Delta}{2} 2^{Q} \\ \frac{\Delta}{2}\left(2^{Q}-1\right) & \text { if } r \geq \frac{\Delta}{2} 2^{Q} .\end{cases}
$$

Here, $\Delta$ is the step size of the quantizer. With a slight abuse of notation, for a complex-valued input $z$, we let $\mathcal{Q}(z)=$ $\mathcal{Q}(\Re\{z\})+j \mathcal{Q}(\Im\{z\})$. Also, it will be convenient to apply $\mathcal{Q}(\cdot)$ element-wise to vector-valued inputs.

We assume LOS propagation conditions and that the antenna elements at the BS are arranged in a ULA. Let $\mathbf{H}=$ $\left[\mathbf{h}_{1}, \ldots \mathbf{h}_{U}\right] \in \mathbb{C}^{B \times U}$ denote the channel matrix, which is known perfectly to the BS. By assuming free-space path loss and by using the plane-wave approximation, we have

$$
\mathbf{h}_{u}=\gamma_{u} \exp \left(-j 2 \pi \epsilon_{\text {sep }} \cos \left(\phi_{u}\right)[0,1, \ldots, B-1]^{T}\right) .
$$

Here, $\gamma_{u}^{2}=\left(\delta_{\text {avg }} / \delta_{u}\right)^{2}$ is the path loss from the BS to the $u$ th $\mathrm{UE}(u=1,2, \ldots, U)$, where $\delta_{u} \in\left(\delta_{\min }, \delta_{\max }\right)$ is the distance (in meters) from the BS to the $u$ th UE and $\delta_{\text {avg }}$ is a normalization parameter. Also, $\epsilon_{\text {sep }}=\delta_{\text {sep }} / \lambda_{c}$, where $\lambda_{c}$ is the carrier wavelength, and $\delta_{\text {sep }} \ll \delta_{\min }$ is the distance (in meters) between two neighboring antenna elements in the ULA. Finally, $\phi_{u} \in\left(-60^{\circ}, 60^{\circ}\right)$ is the azimuth angle to the $u$ th UE ( $u=1,2, \ldots, U)$ measured from the boresight of the ULA.

\section{UPLINK TRANSMISSION}

The $B$-dimensional complex-valued received signal at the output of the ADCs at the BS is

$$
\mathbf{y}^{\mathrm{ul}}=\mathcal{Q}\left(\mathbf{A}\left(\mathbf{H s}^{\mathrm{ul}}+\mathbf{n}^{\mathrm{ul}}\right)\right) .
$$

Here, $\mathbf{s}^{\mathrm{ul}}=\left[s_{1}^{\mathrm{ul}}, s_{2}^{\mathrm{ul}}, \ldots, s_{U}^{\mathrm{ul}}\right]^{T} \in \mathbb{C}^{U}$ contains the information symbols from the $U$ UEs, which satisfy the power constraint $\mathbb{E}\left[\left|s_{u}^{\mathrm{ul}}\right|^{2}\right] \leq 1, u=1,2, \ldots, U$. Furthermore, $\mathbf{n}^{\mathrm{ul}} \sim$ $\mathcal{C N}\left(\mathbf{0}_{B}, N_{0}^{\mathrm{ul}} \mathbf{I}_{B}\right)$ is the additive noise at the BS. The diagonal matrix $\mathbf{A} \in \mathbb{R}^{B \times B}$ models the amplification gain set by the automatic gain control circuit. The aim of this circuit is to scale the received signal so that it matches the dynamic range of the ADCs, which depends on the number of bits $Q$ and on the step size $\Delta$ (see (2)). Specifically, we assume that

$$
\mathbf{A}=\sqrt{1 / B} \operatorname{diag}\left(\mathbf{H H}^{H}+N_{0}^{\mathrm{ul}} \mathbf{I}_{B}\right)^{-1 / 2}
$$

which ensures that the average power of the received signal at each antenna is $1 / B$ prior to quantization.

We use Bussgang's theorem [21] to characterize the impact of low-precision quantization on massive MIMO performance as first done in [22] and then in, e.g., [3,4,9,11,23,24]. This theorem states that the correlation of two Gaussian signals, taken after one of them is passed through a nonlinear distortion, is proportional to the correlation computed before the nonlinear distortion. Bussgang's theorem enables us to linearize the uplink channel input-output relation (4) as

$$
\mathbf{y}^{\mathrm{ul}}=\mathbf{G}^{\mathrm{ul}} \mathbf{A}\left(\mathbf{H s}^{\mathrm{ul}}+\mathbf{n}^{\mathrm{ul}}\right)+\mathbf{e}^{\mathrm{ul}} .
$$

Here, $\mathrm{e}^{\mathrm{ul}} \in \mathbb{C}^{B}$ is a non-Gaussian additive quantization noise that is uncorrelated with $\mathbf{s}^{\mathrm{ul}}$. Furthermore, we have [3]

$$
\begin{aligned}
\mathbf{G}^{\mathrm{ul}} & =\frac{\Delta}{\sqrt{\pi}} \operatorname{diag}\left(\mathbf{C}_{\mathbf{z}}^{\mathrm{ul}}\right)^{-1 / 2} \\
& \times \sum_{i=1}^{2^{Q}-1} \exp \left(-\frac{\Delta^{2}}{2}\left(i-2^{Q-1}\right)^{2} \operatorname{diag}\left(\mathbf{C}_{\mathbf{z}}^{\mathrm{ul}}\right)^{-1}\right)
\end{aligned}
$$

where $\mathbf{C}_{\mathbf{z}}^{\mathrm{ul}}=\mathbf{A}\left(\mathbf{H H}^{H}+N_{0}^{\mathrm{ul}} \mathbf{I}_{B}\right) \mathbf{A}$ is the covariance matrix of the quantizers' input $\mathbf{z}^{\mathrm{ul}}=\mathbf{A}\left(\mathbf{H s}^{\mathrm{ul}}+\mathbf{n}^{\mathrm{ul}}\right)$.

We assume linear detection at the BS. Specifically, a soft estimate $\hat{\mathbf{s}}^{\mathrm{ul}} \in \mathbb{C}^{U}$ of $\mathbf{s}^{\mathrm{ul}}$ is obtained at the BBU as follows:

$$
\hat{\mathbf{s}}^{\mathrm{ul}}=\mathbf{W} \mathbf{y}^{\mathrm{ul}}=\mathbf{W} \mathcal{Q}\left(\mathbf{A}\left(\mathbf{H s}^{\mathrm{ul}}+\mathbf{n}^{\mathrm{ul}}\right)\right) .
$$

Here, $\mathbf{W} \in \mathbb{C}^{U \times B}$ is a linear filter. We consider matched filtering (MF) and zero forcing (ZF), for which we have

$$
\mathbf{W}= \begin{cases}\left(\mathbf{G}^{\mathrm{ul}} \mathbf{A} \mathbf{H}\right)^{H}, & \text { for } \mathrm{MF}, \\ \left(\left(\mathbf{G}^{\mathrm{ul}} \mathbf{A} \mathbf{H}\right)^{H} \mathbf{G}^{\mathrm{ul}} \mathbf{A} \mathbf{H}\right)^{-1}\left(\mathbf{G}^{\mathrm{ul}} \mathbf{A} \mathbf{H}\right)^{H}, & \text { for } \mathbf{Z F} .\end{cases}
$$


Using (6) and (8), we write the uplink signal-to-interferencenoise-and-distortion ratio (SINDR) at the $u$ th UE as

$$
\gamma_{u}^{\mathrm{ul}}=\frac{\left|\mathbf{w}_{u}^{T} \mathbf{G}^{\mathrm{ul}} \mathbf{A} \mathbf{h}_{u}\right|^{2}}{\sum_{v \neq u}\left|\mathbf{w}_{u}^{T} \mathbf{G}^{\mathrm{ul}} \mathbf{A} \mathbf{h}_{v}\right|^{2}+\mathbf{w}_{u}^{T} \mathbf{C}_{\mathbf{e}}^{\mathrm{ul}} \mathbf{w}_{u}^{*}+N_{0}^{\mathrm{ul}}\left\|\mathbf{A} \mathbf{G}^{\mathrm{ul}} \mathbf{w}_{u}\right\|^{2}} .
$$

Here, $\mathbf{w}_{u} \in \mathbb{C}^{B}$ is the $u$ th column of $\mathbf{W}^{T}$. To compute (10), we need the covariance matrix $\mathbf{C}_{\mathbf{e}}^{\mathrm{ul}}=\mathbb{E}\left[\mathbf{e}^{\mathrm{ul}}\left(\mathbf{e}^{\mathrm{ul}}\right)^{H}\right]$ of the quantization noise $\mathbf{e}^{\mathrm{ul}}$. When $Q=1$, the matrix $\mathbf{C}_{\mathbf{e}}^{\text {ul }}$ can be computed in closed form using Van Vleck's arcsine law $[22,25]$. Unfortunately, no closed-form expression for $\mathbf{C}_{\mathrm{e}}^{\mathrm{ul}}$ is available for $Q>1$. In the numerical results in Section 6, we evaluate $\mathbf{C}_{\mathbf{e}}^{\mathrm{ul}}$ for the case $Q>1$ using the diagonal approximation put forward in [17, Sec. IV], which involves approximating $\mathbf{C}_{\mathbf{e}}^{\mathrm{ul}}$ as

$$
\begin{aligned}
& \mathbf{C}_{\mathbf{e}}^{\mathrm{ul}} \approx \frac{\Delta^{2}}{2}\left(2^{Q}-1\right)^{2} \mathbf{I}_{B}-\mathbf{G}^{\mathrm{ul}} \operatorname{diag}\left(\mathbf{C}_{\mathbf{z}}^{\mathrm{ul}}\right) \mathbf{G}^{\mathrm{ul}}-4 \Delta^{2} \\
& \times \sum_{i=1}^{2^{Q}-1}\left(i-2^{Q-1}\right) \Phi\left(\sqrt{2}\left(i-2^{Q-1}\right) \operatorname{diag}\left(\mathbf{C}_{\mathbf{z}}^{\mathrm{ul}}\right)^{-1 / 2}\right) .
\end{aligned}
$$

Here, $\Phi(x)=\frac{1}{2 \pi} \int_{-\infty}^{x} e^{-u^{2} / 2} \mathrm{~d} u$ is the cumulative distribution function of the standard normal random variable. This approximation turns out to be accurate for $Q \geq 3$.

\section{DOWNLINK TRANSMISSION}

The received signals at the $U$ UEs are given by

$$
\mathbf{y}^{\mathrm{dl}}=\mathbf{H}^{T} \mathcal{Q}\left(\mathbf{P s}^{\mathrm{dl}}\right)+\mathbf{n}^{\mathrm{dl}} .
$$

Here, the vector $\mathbf{n}^{\mathrm{dl}} \sim \mathcal{C N}\left(\mathbf{0}_{U}, N_{0}^{\mathrm{dl}} \mathbf{I}_{U}\right)$ denotes the additive noise at the UEs and $\mathbf{s}^{\mathrm{dl}}=\left[s_{1}^{\mathrm{dl}}, s_{2}^{\mathrm{dl}}, \ldots, s_{U}^{\mathrm{dl}}\right]^{T} \in \mathbb{C}^{U}$ contains the information symbols intended to the $U$ UEs, which satisfy $\mathbb{E}\left[\left|s_{u}^{\mathrm{dl}}\right|^{2}\right]=1, u=1, \ldots, U$. We assume linear precoding at the BS. Specifically, prior to quantization, the information symbols $\mathbf{s}^{\mathrm{dl}}$ are mapped to the BS antenna array through multiplication by a precoding matrix $\mathbf{P} \in \mathbb{C}^{B \times U}$. We consider $\mathrm{MF}$ and ZF precoding, for which we have

$$
\mathbf{P}= \begin{cases}\left(1 / \beta^{\mathrm{MF}}\right) \mathbf{H}^{*}, & \text { for } \mathrm{MF} \\ \left(1 / \beta^{\mathrm{ZF}}\right) \mathbf{H}^{*}\left(\mathbf{H}^{T} \mathbf{H}^{*}\right)^{-1}, & \text { for } \mathrm{ZF}\end{cases}
$$

The constants $\beta^{\mathrm{MF}}$ and $\beta^{\mathrm{ZF}}$ ensure that the power constraint $\mathbb{E}\left[\left\|\mathbf{P s}^{\mathrm{dl}}\right\|^{2}\right]=1$ is satisfied. To match the discrete levels supported by the DACs, the precoded vector $\mathbf{P} \mathbf{s}^{\mathrm{dl}}$ is quantized using (2), prior to transmission over the fronthaul link.

As in the uplink case, we linearize the downlink channel input-output relation (12) using Bussgang's theorem as [11]

$$
\mathbf{y}^{\mathrm{dl}}=\mathbf{H}^{T}\left(\mathbf{G}^{\mathrm{dl}} \mathbf{P} \mathbf{s}^{\mathrm{dl}}+\mathbf{e}^{\mathrm{dl}}\right)+\mathbf{n}^{\mathrm{dl}} .
$$

Here, $\mathbf{e}^{\mathrm{dl}} \in \mathbb{C}^{B}$ is a non-Gaussian additive quantization noise that is uncorrelated with $\mathbf{s}^{\mathrm{dl}}$. Furthermore, $\mathbf{G}^{\mathrm{dl}}$ can be obtained from (7) by replacing $\mathbf{C}_{\mathbf{z}}^{\mathrm{ul}}$ by $\mathbf{C}_{\mathbf{z}}^{\mathrm{dl}}=\mathbf{P} \mathbf{P}^{H}$, which is the covariance matrix of the quantizer's input $\mathbf{z}^{\mathrm{dl}}=\mathbf{P s}^{\mathrm{dl}}$. We assume that, at the $u$ th UE, a soft estimate $\hat{s}_{u}^{\mathrm{dl}}$ of $s_{u}^{\mathrm{dl}}$ is obtained as $\hat{s}_{u}^{\mathrm{dl}}=\beta_{u} y_{u}^{\mathrm{dl}}$, where $\beta_{u}=\left(\left[\mathbf{H}^{T} \mathbf{G}^{\mathrm{dl}} \mathbf{P}\right]_{u, u}\right)^{-1}$. This requires that $\beta_{u}$ is known at the $u$ th UE. We now use (14) to compute the downlink SINDR for the $u$ th UE as

$$
\gamma_{u}^{\mathrm{dl}}=\frac{\left|\mathbf{h}_{u}^{T} \mathbf{G}^{\mathrm{dl}} \mathbf{p}_{u}\right|^{2}}{\sum_{v \neq u}\left|\mathbf{h}_{u}^{T} \mathbf{G}^{\mathrm{dl}} \mathbf{p}_{v}\right|^{2}+\mathbf{h}_{u}^{T} \mathbf{C}_{\mathbf{e}}^{\mathrm{dl}} \mathbf{h}_{u}^{*}+N_{0}^{\mathrm{dl}}} .
$$

Here, $\mathbf{h}_{u}$ and $\mathbf{p}_{u}$ is the $u$ th column of $\mathbf{H}$ and $\mathbf{P}$, respectively. For $Q=1$, we evaluate the covariance matrix $\mathbf{C}_{\mathbf{e}}^{\mathrm{dl}}$ of the quantization noise $\mathbf{e}^{\mathrm{dl}}$ using Van Vleck's arcsine law [22,25]. For $Q>1$, we use the diagonal approximation in (11) with $\mathbf{C}_{\mathbf{z}}^{\mathrm{ul}}$ replaced by $\mathbf{C}_{\mathbf{z}}^{\mathrm{dl}}$.

\section{ACHIEVABLE RATES}

In our channel model, $\mathbf{H}$ is a deterministic function of the UEs' positions. Assuming that the UEs' positions are fixed for the duration of each codeword and that $\mathbf{H}$ is known at the BS, we can lower-bound the uplink rate of the $u$ th UE as

$$
R_{u}^{\mathrm{ul}}=\log _{2}\left(1+\gamma_{u}^{\mathrm{ul}}\right)
$$

where the uplink SINDR $\gamma_{u}^{\text {ul }}$ is given in (10). This rate is achievable using a Gaussian codebook and a scaled nearestneighbor detection rule at the BS [24,26,27]. Similarly, the downlink rate $R_{u}^{\mathrm{ul}}$ for the $u$ th UE, for a given UEs' placement, is lower-bounded by

$$
R_{u}^{\mathrm{dl}}=\log _{2}\left(1+\gamma_{u}^{\mathrm{dl}}\right)
$$

where the downlink SINDR $\gamma_{u}^{\mathrm{dl}}$ is given in (15).

For the case in which the UEs' placement is random but remains constant for the duration of each codeword, it is natural to consider as performance metric the uplink and downlink outage probabilities, which are given by

$$
P_{\text {out }}^{\mathrm{k}}\left(R_{\text {sum }}^{\mathrm{k}}\right)=\mathbb{P}\left[\sum_{u=1}^{U} R_{u}^{\mathrm{k}}<R_{\text {sum }}^{\mathrm{k}}\right]
$$

where $R_{\text {sum }}^{\mathrm{k}}$ is the target sum rate and $\mathrm{k} \in\{\mathrm{ul}, \mathrm{dl}\}$.

\section{NUMERICAL RESULTS}

We now present numerical simulation results to investigate how the uplink rate (16) and the downlink rate (17) depend on the precision of the ADCs and DACs and on the number of antenna elements, for a given fronthaul-bandwidth constraint and for a given constraint on the length of the ULA. Specifically, we assume that the system operates at $f_{c}=30 \mathrm{GHz}$. Hence, the carrier wavelength is $\lambda_{c}=0.01$ meters. The step size $\Delta$ of the quantizers is chosen as a function of the number of BS antennas $B$ and of the number of bits $Q$ of the quantizer 


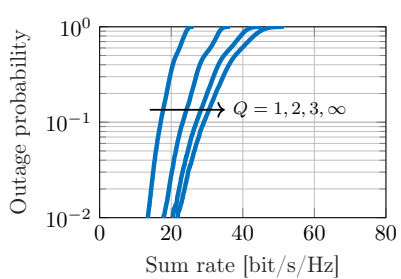

(a) Uplink: MF and $\gamma^{\mathrm{ul}}=0 \mathrm{~dB}$.

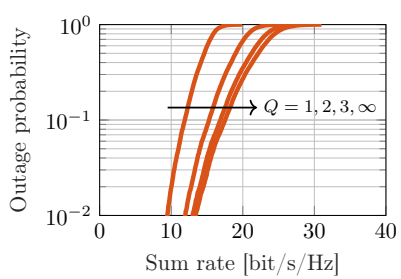

(c) Downlink: MF and $\gamma^{\mathrm{dl}}=0 \mathrm{~dB}$.

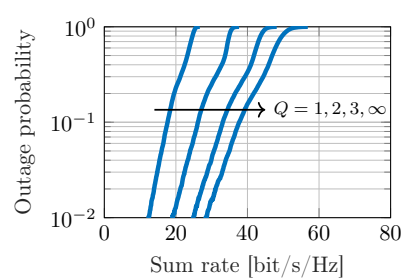

(b) Uplink: ZF and $\gamma^{\mathrm{ul}}=0 \mathrm{~dB}$.

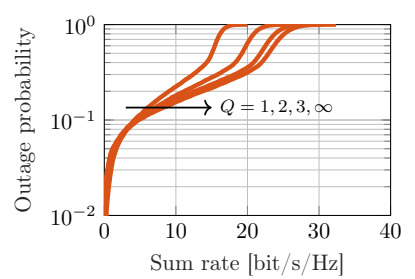

(d) Downlink: ZF and $\gamma^{\mathrm{dl}}=0 \mathrm{~dB}$.
Fig. 2. Uplink and downlink outage probabilities for different values of $Q ; B=64, U=8$, and $\epsilon_{\text {sep }}=0.5$. Here, there is no constraint on the fronthaul rate $R^{\text {fh }}=2 B Q$.

as $\Delta=2^{-Q+1}(2 B)^{-1 / 2}\left(1-\Phi\left(10^{-3} / 2\right)\right)$. With this choice, the probability that the input to the quantizer is clipped (i.e., it exceeds the clipping level $2^{Q-1} \Delta$ ), when its variance is $1 / B$, is $10^{-3}$. We assume that $U=8$ UEs are dropped randomly according to a uniform distribution over a section of a circular ring with inner radius $\delta_{\min }=50$ meters, outer radius $\delta_{\max }=150$ meters, and azimuth angle $\phi_{u} \in\left(-60^{\circ}, 60^{\circ}\right)$. We also set $\delta_{\text {avg }}=\frac{2}{3}\left(\delta_{\max }^{3}-\delta_{\min }^{3}\right) /\left(\delta_{\max }^{2}-\delta_{\min }^{2}\right) \approx 108$ meters to be the average distance between the BS and a UE in the cell.

In Fig. 2, we plot the uplink and downlink outage probabilities, evaluated by simulating $10^{4}$ random UE placements. Here, $B=64, \epsilon_{\text {sep }}=0.5, Q \in\{1,2,3, \infty\}$, and there is no constraint on the fronthaul rate (1). We also let $\rho^{\mathrm{ul}}=1 / N_{0}^{\mathrm{ul}}$ and $\rho^{\mathrm{dl}}=1 / N_{0}^{\mathrm{dl}}$ denote the uplink and downlink SNR, respectively. Note that by increasing the number of bits, we approach the rate achievable with infinite-precision data converters; this behavior holds for the uplink as well as the downlink, and for both MF and ZF.

Next, we show in Fig. 3 uplink and downlink rates with MF, evaluated again by simulating $10^{4}$ random UE placements, for the case when the system is subject to a fronthaul-bandwidth constraint. Specifically, we plot the uplink sum rate $R_{\text {sum }}^{\mathrm{ul}}$ corresponding to $P_{\text {out }}^{\mathrm{ul}}=10 \%$ as a function of the precision of the ADCs. We present a similar plot for the downlink, where now $Q$ indicates the precision of the DACs. The available fronthaul bandwidth is fixed to $R^{\mathrm{fh}}=512 \mathrm{bit} / \mathrm{s} / \mathrm{Hz}$ and the length of the ULA is fixed to $L_{\mathrm{ULA}}=128 \lambda_{c}=1.28$ meters. The number of BS antennas $B$ is obtained from the precision $Q$ of the quantizers and from the fronthaul bandwidth $R^{\text {fh }}$ as $B=\left\lfloor R^{\mathrm{fh}} /(2 Q)\right\rfloor$. Furthermore, the antenna separation is $\epsilon_{\mathrm{sep}}=L_{\mathrm{ULA}} /\left(B \lambda_{c}\right)$.

We observe that the optimal number of bits depends on

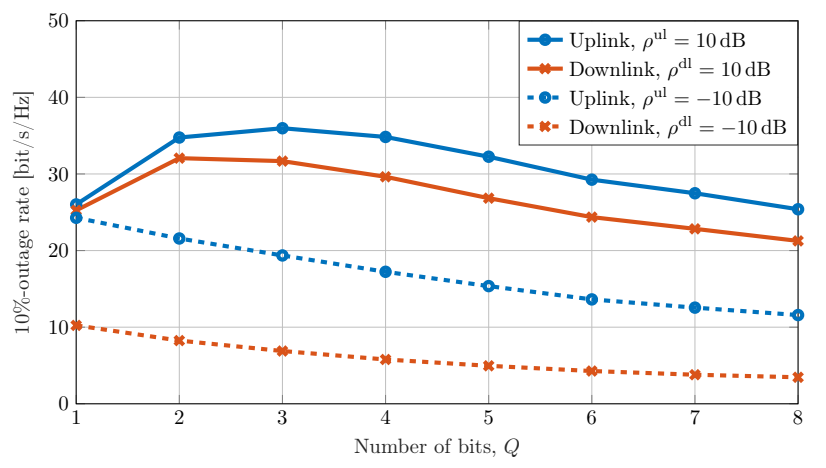

Fig. 3. Uplink and downlink outage rates for different values of $Q ; L_{\mathrm{ULA}}=128 \lambda_{c}=1.28$ meters, $R^{\mathrm{fh}}=512 \mathrm{bit} / \mathrm{s} / \mathrm{Hz}$, $B=\left\lfloor R^{\mathrm{fh}} /(2 Q)\right\rfloor$, and $\epsilon_{\mathrm{sep}}=L_{\mathrm{ULA}} /\left(B \lambda_{c}\right)$.

the SNR operating point. For example, in the uplink and for $\rho^{\mathrm{ul}}=-10 \mathrm{~dB}$, we find that setting $Q=1$ (which gives $B=256$ antennas and $\epsilon_{\text {sep }}=0.5$ ) yields the highest sum rate. In contrast, for $\rho^{\mathrm{ul}}=10 \mathrm{~dB}$, setting $Q=3$ (which corresponds to $B=85$ antennas and $\epsilon_{\mathrm{sep}}=1.5$ ) yields the highest uplink sum rate. The reason is that at low SNR, the additive noise at the BS dominates over the quantization error introduced by the low-precision converters. Hence, it is better to have a large number of antennas connected to lowprecision ADCs, because this maximizes the array gain. The opposite holds true at high SNR. In this case, it is better to use a lower number of antennas connected to higher-precision ADCs. This yields a better linearity in the system and allows the BS to separate the UEs through simple linear-processing techniques. Similar considerations hold for the downlink.

\section{CONCLUSIONS AND EXTENSIONS}

We have investigated how the achievable uplink and downlink rates in all-digital massive MIMO architectures depend on the precision of the data converters at the BS and on the number of antenna elements, given a constraint on the fronthaul bandwidth and on the length of the antenna array. Our results illustrate that, at low SNR, it is preferable to equip the BS with many antenna elements connected to low-precision converters, whereas at high SNR it is better to have fewer antenna elements connected to higher-precision converters.

It is worth highlighting that our investigation is limited to a restricted number of system parameters and a simple LOS channel model. Also, it pertains a simplified scenario in which the fronthaul link carries uncompressed data. By moving some signal-processing capabilities (e.g., linear detection/precoding) from the BBU to the RRH and/or by compressing the data prior to fronthaul transmission, one could reduce significantly the required fronthaul bandwidth. This would enable the use of higher-precision converters in systems with a larger number of active antenna elements. Such extensions will be considered in future work. 


\section{REFERENCES}

[1] C. Risi, D. Persson, and E. G. Larsson, "Massive MIMO with 1-bit ADC," Apr. 2014. [Online]. Available: http://arxiv.org/abs/1404.7736

[2] J. Zhang, L. Dai, S. Sun, and Z. Wang, "On the spectral efficiency of massive MIMO systems with lowresolution ADCs," IEEE Commun. Lett., vol. 20, no. 5, pp. $842-845$, Dec. 2015.

[3] S. Jacobsson, G. Durisi, M. Coldrey, U. Gustavsson, and C. Studer, "Throughput analysis of massive MIMO uplink with low-resolution ADCs," IEEE Trans. Wireless Commun., vol. 16, no. 6, pp. 4038-4051, Jun. 2017.

[4] Y. Li, C. Tao, G. Seco-Granados, A. Mezghani, A. L. Swindlehurst, and L. Liu, "Channel estimation and performance analysis of one-bit massive MIMO systems," IEEE Trans. Signal Process., vol. 65, no. 15, pp. 40754089, Aug. 2017.

[5] J. Choi, J. Mo, and R. W. Heath Jr., "Near maximumlikelihood detector and channel estimator for uplink multiuser massive MIMO systems with one-bit ADCs," IEEE Trans. Commun., vol. 64, no. 5, pp. 2005-2018, May 2016.

[6] C.-K. Wen, C.-J. Wang, S. Jin, K.-K. Wong, and P. Ting, "Bayes-optimal joint channel-and-data estimation for massive MIMO with low-precision ADCs," IEEE Trans. Signal Process., vol. 64, no. 10, pp. 2541-2556, Jul. 2015.

[7] C. Mollén, J. Choi, E. G. Larsson, and R. W. Heath Jr., "Uplink performance of wideband massive MIMO with one-bit ADCs," IEEE Trans. Wireless Commun., vol. 16, no. 1 , pp. 87-100, Oct. 2016.

[8] C. Studer and G. Durisi, "Quantized massive MUMIMO-OFDM uplink," IEEE Trans. Commun., vol. 64, no. 6, pp. 2387-2399, Jun. 2016.

[9] Y. Li, C. Tao, A. L. Swindlehurst, A. Mezghani, and L. Liu, "Downlink achievable rate analysis in massive MIMO systems with one-bit DACs," IEEE Commun. Lett., vol. 21, no. 7, pp. 1669-1672, Jul. 2017.

[10] A. K. Saxena, I. Fijalkow, and A. L. Swindlehurst, "Analysis of one-bit quantized precoding for the multiuser massive MIMO downlink," IEEE Trans. Signal Process., vol. 65, no. 17, pp. 4624-4634, Sep. 2017.

[11] S. Jacobsson, G. Durisi, M. Coldrey, T. Goldstein, and C. Studer, "Quantized precoding for massive MUMIMO," IEEE Trans. Commun., vol. 65, no. 11, pp. 4670-4684, Nov. 2017.

[12] H. Jedda, J. A. Nossek, and A. Mezghani, "Minimum BER precoding in 1-bit massive MIMO systems," in IEEE Sensor Array and Multichannel Signal Process. Workshop (SAM), Rio de Janeiro, Brazil, Jul. 2016.

[13] L. Landau and R. C. de Lamare, "Branch-and-bound precoding for multiuser MIMO systems with 1-bit quantization," IEEE Wireless Commun. Lett., vol. 6, no. 6, pp. 770-773, Dec. 2017.
[14] O. Castañeda, S. Jacobsson, G. Durisi, M. Coldrey, T. Goldstein, and C. Studer, "1-bit massive MU-MIMO precoding in VLSI," IEEE J. Emerging Sel. Topics Circuits Syst., vol. 7, no. 4, pp. 508-522, Dec. 2017.

[15] A. L. Swindlehurst, A. K. Saxena, A. Mezghani, and I. Fijalkow, "Minimum probability-of-error perturbation precoding for the one-bit massive MIMO downlink," in Proc. IEEE Int. Conf. Acoust., Speech, Signal Process. (ICASSP), New Orleans, LA, USA, Mar. 2017, pp. 6483-6487.

[16] A. Nedelcu, F. Steiner, M. Staudacher, G. Kramer, W. Zirwas, R. Sisava Ganesan, P. Baracca, and S. Wesemann, "Quantized precoding for multi-antenna downlink channels with MAGIQ," in Int. ITG Workshop on Smart Antennas (WSA), Bochum, Germany, Mar. 2017.

[17] S. Jacobsson, G. Durisi, M. Coldrey, and C. Studer, "Linear precoding with low-resolution DACs for massive MU-MIMO-OFDM downlink," Sep. 2017. [Online]. Available: https://arxiv.org/abs/1709.04846

[18] S. Jacobsson, O. Castañeda, C. Jeon, G. Durisi, and C. Studer, "Nonlinear precoding for phase-quantized constant-envelope massive MU-MIMO-OFDM," in IEEE Int. Conf. Telecommunications, Saint-Malo, France, Jun. 2018, to appear.

[19] S.-H. Park, O. Simeone, O. Sahin, and S. Shamai (Shitz), "Fronthaul compression for cloud radio access networks: Signal processing advances inspired by network information theory," IEEE Signal Process. Mag., vol. 31, no. 6, pp. 69-79, Nov. 2014.

[20] M. Peng, C. Wang, V. Lau, and H. V. Poor, "Fronthaulconstrained cloud radio access networks: insights and challenges," IEEE Wireless Commun., vol. 22, no. 2, pp. 152-160, Apr. 2015.

[21] J. J. Bussgang, "Crosscorrelation functions of amplitude-distorted Gaussian signals," Res. Lab. Elec., Cambridge, MA, Tech. Rep. 216, Mar. 1952.

[22] A. Mezghani and J. A. Nossek, "Capacity lower bound of MIMO channels with output quantization and correlated noise," in IEEE Int. Symp. Inf. Theory (ISIT), Cambridge, MA, USA, Jul. 2012.

[23] A. Mezghani and A. L. Swindlehurst, "mmWave massive MIMO with simple RF and appropriate DSP," in Proc. Asilomar Conf. Signals, Syst., Comput., Pacific Grove, CA, USA, Oct.-Nov. 2017, pp. 277-284.

[24] B. Li, L. N., and W. Zhang, "On transmission model for massive MIMO under low-resolution output quantization," in Proc. IEEE Veh. Technol. Conf. Spring (VTCSpring), Sydney, Australia, Jun. 2017.

[25] J. H. Van Vleck and D. Middleton, "The spectrum of clipped noise," Proc. IEEE, vol. 54, no. 1, pp. 2-19, Jan. 1966.

[26] A. Lapidoth and S. Shamai (Shitz), "Fading channels: How perfect need "perfect side information" be?" IEEE Trans. Inf. Theory, vol. 48, no. 5, pp. 1118-1134, May 2002.

[27] W. Zhang, "A general framework for transmission with transceiver distortion and some applications," IEEE Trans. Commun., vol. 60, no. 2, pp. 384-399, Feb. 2012. 